\title{
An E3 Ubiquitin Ligase, Synoviolin, Is Involved in the Degradation of Homocysteine-Inducible Endoplasmic Reticulum Protein
}

\author{
Tomoji Maeda, ${ }^{*, a \dagger}$ Yu Fujita, ${ }^{a}$ Chiaki Tanabe-Fujimura, ${ }^{a}$ Kun Zou, ${ }^{a}$ Junjun Liu, ${ }^{a}$ Shuyu Liu, ${ }^{a}$ \\ Kota Kikuchi, ${ }^{a}$ Xuefeng Shen,${ }^{a}$ Toshihiro Nakajima, ${ }^{b}$ and Hiroto Komano*,a \\ ${ }^{a}$ Department of Neuroscience, School of Pharmacy, Iwate Medical University; 2-1-1 Nishitokuta, Yahaba, Iwate \\ 028-3694, Japan: and ${ }^{b}$ Institute of Medical Science, Tokyo Medical University; 6-1-1 Shinjuku, Shinjyuku-ku, Tokyo \\ 160-8402, Japan.
}

Received January 11, 2018; accepted March 20, 2018

\begin{abstract}
Homocysteine-inducible endoplasmic reticulum (ER) protein (Herp) is an ER stress-inducible membrane protein involved in ER-associated degradation. Herp expression is maintained at low levels through a strict regulatory mechanism, but the details of this mechanism and the reasons why Herp expression is restricted in this manner remain unclear. Here, we show that Herp degradation involves synoviolin, an ER-resident E3 ubiquitin ligase. Herp protein levels were found to be markedly elevated in synoviolin-null cells, and Herp expression decreased when synoviolin was overexpressed. However, the lysine residues of Herp, which are ubiquitinated by E3 ubiquitin ligase, were not sufficient for regulation of Herp degradation. These results suggest that Herp degradation is mediated via synoviolin and that Herp ubiquitination involves amino acids other than lysine.
\end{abstract}

Key words homocysteine-inducible endoplasmic reticulum protein; synoviolin; ubiquitin; proteasome; endoplasmic reticulum

Homocysteine-inducible endoplasmic reticulum (ER) protein (Herp) is strongly upregulated by the unfolded protein response, which plays a pivotal role in cellular homeostasis by expanding the protein-folding capacity of the ER to cope with unfolded or misfolded proteins. ${ }^{1-3)}$ Herp has been shown to be involved in the turnover of nonglycosylated BiP substrates $^{4)}$ in ER-associated protein degradation (ERAD) and the $N$-glycan-independent ERAD pathway, ${ }^{5)}$ and exerts antiapoptotic effects in response to ER stress. ${ }^{3)}$ Moreover, we have previously demonstrated that the ubiquitin-like domain (ULD) of Herp affects the regulation of intracellular Herp levels during ER stress. ${ }^{6)}$ Recently, we also reported that Herp is likely to be involved in the degradation of immature nicastrin and other excess components of the $\gamma$-secretase complex, suggesting that Herp plays a role in eliminating excess unassembled components of multimeric complexes. ${ }^{7)}$

Herp associates with high-molecular-mass protein complexes containing derlin-1, p97, and the E3 ubiquitin-protein ligase synoviolin/Hrd1, which mediates the ubiquitination and retrotranslocation of substrate proteins to the cytosol. ${ }^{2}$ In addition, Herp can bind to ubiquitinated proteins and the $26 \mathrm{~S}$ proteasome. ${ }^{4,8)}$ Ubiquitination is required for proteasomemediated degradation; however, recent evidence has shown that ubiquitin serves multiple additional functions, including in intracellular trafficking. ${ }^{9)}$ Synoviolin, a representative ER-resident E3 ubiquitin ligase, is a mammalian homolog of yeast $\mathrm{Hrd1}^{2}{ }^{2}$ Synoviolin is also a known pathogenic factor in rheumatoid arthritis ${ }^{10)}$ and is involved in liver cirrhosis ${ }^{11)}$ and ERAD. Substrates of this enzyme include the tumorsuppressor protein $\mathrm{p} 53^{12)}$ and nuclear factor E2-related factor-2 (Nrf2), a transcription factor that regulates cellular redox ho-

\footnotetext{
†Present address: Department of Pharmacology, Nihon Pharmaceutical University; 10281 Komuro, Ina-machi, Kitaadachi-gun, Saitama 362-0806, Japan.
}

meostasis. $^{11)}$

Herp is a short-lived protein with a half-life of approximately $2 \mathrm{~h}$ that is regulated by the ubiquitin-dependent and -independent proteasome pathways. ${ }^{6,13)}$ Herp is thought to bind to synoviolin and promote its E3 ubiquitination activity, thereby acting as a regulator of synoviolin-dependent protein degradation. ${ }^{14)}$ However, the mechanisms regulating Herp degradation and the reasons underlying its tight regulation remain unknown. Therefore, in the present study, we sought to determine whether synoviolin-mediated proteasomal degradation is involved in this process.

\section{MATERIALS AND METHODS}

Antibodies, Reagents, and Cell Lines Affinity-purified rabbit anti-Herp antibodies were prepared as previously described. ${ }^{1)}$ Anti-BiP, anti-synoviolin, and anti-green fluorescent protein (GFP) antibodies were purchased from Invitrogen (Carlsbad, CA, U.S.A.). Anti- $\alpha$-tubulin antibody was purchased from Santa Cruz Biotechnology, Inc. (Santa Cruz, CA, U.S.A.). Anti-FLAG antibody and MG132 were purchased from Sigma (St. Louis, MO, U.S.A.). Anti-ubiquitin antibody was purchased from BIOMOL (Plymouth Meeting, PA, U.S.A.). Synoviolin-wild-type (wt) and synoviolin-null mouse embryonic fibroblasts ${ }^{15)}$ were cultured in Dulbecco's modified Eagle's medium (Wako Pure Chemical Industries, Ltd., Osaka, Japan) containing 10\% fetal calf serum (Invitrogen).

Plasmids and Retroviral Transduction pMX-Herp, pMX- $\Delta$ Ub-Herp, pcDNA3.1-Herp-FLAG (C-terminal FLAGtagged Herp), and pcDNA3.1- $\Delta$ Ub-Herp-FLAG (C-terminal FLAG-tagged $\Delta \mathrm{Ub}$-Herp) constructs were generated as previously described.' ${ }^{7)}$ pMX- $\Delta$ Ub-Herp4KR and pMX-Herp9KR were constructed by TaKaRa Bio Inc. (Shiga, Japan) by twostep PCR mutagenesis involving mutation of the four lysine residues downstream of the ULD (positions 89, 93, 100, 375) 
(A)

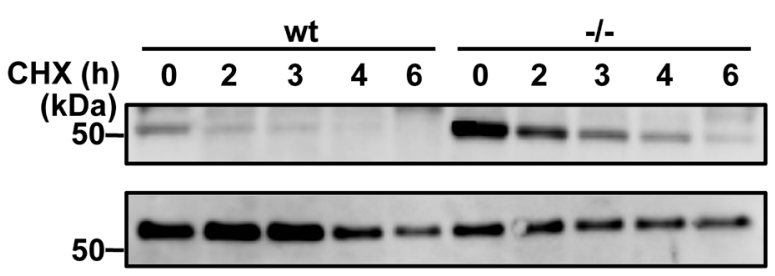

Herp

$\alpha-$ Tub

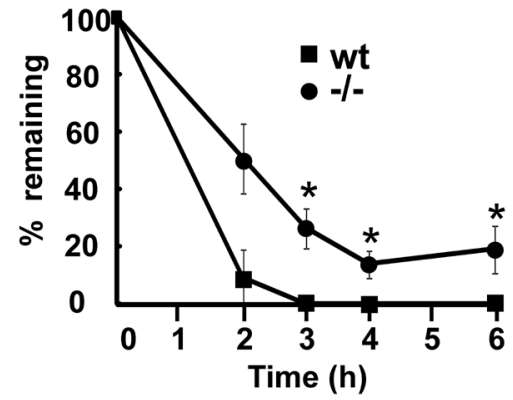

(B)

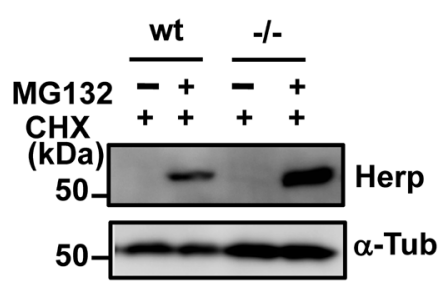

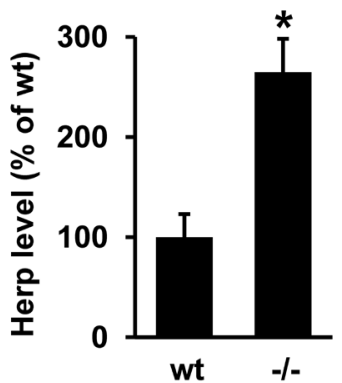

Fig. 1. Herp Accumulation in Synoviolin-Null Cells

(A) Synoviolin-null and wt cells were treated with $20 \mu \mathrm{g} / \mathrm{mL}$ cycloheximide and collected at the indicated times. Herp in RIPA-solubilized lysates (20 $\mu \mathrm{g})$ was then detected by immunoblotting using an anti-Herp antibody. $\alpha$-Tubulin levels in the lysates were used as an internal loading control. Each sample was run in duplicate. The intensities of the bands corresponding to Herp in the left panel were densitometrically quantified using an LAS-3000 luminescent image analyzer. Herp levels at each time point were calculated as a percentage of the band intensity at $0 \mathrm{~h}$. Values are expressed as the mean \pm standard error (S.E.) of four independent experiments. $* p<0.05$ (Student's $t$-test), synoviolin-null cells $v s$. wt cells. CHX, cycloheximide. (B) Synoviolin-null and wt cells were treated with $20 \mu \mathrm{g} / \mathrm{mL}$ cycloheximide with or without $10 \mu \mathrm{M}$ MG132 for $6 \mathrm{~h}$. Levels of Herp in RIPA-solubilized lysates $(20 \mu \mathrm{g})$ were then measured by immunoblotting with an anti-Herp antibody. The right panel shows Herp levels in cells treated with cycloheximide and MG132 quantified from three independent experiments. Values are expressed as the mean \pm S.E. $* p<0.05$ (two-tailed Student's $t$-test).

and all nine lysine residues in the Herp protein, respectively, to arginine. The plasmids pcDNA-Syvn and pcDNA-Syvn C307A were generated as reported previously. ${ }^{10,16)}$ Retroviral transduction was performed according to published methods. $^{16)}$

Cycloheximide and MG132 Treatments Cycloheximide chase experiments were performed as previously described. ${ }^{7)}$ Cells were plated on $60-\mathrm{mm}$ tissue culture dishes $\left(5 \times 10^{5}\right.$ cells/ dish) and cultured for $24 \mathrm{~h}$, before addition of $20 \mu \mathrm{g} / \mathrm{mL}$ cycloheximide with or without $10 \mu \mathrm{M}$ MG132. At $0,2,3,4$, and $6 \mathrm{~h}$ after cycloheximide treatment and $6 \mathrm{~h}$ after cycloheximide and MG132 treatment, the cells were lysed in radioimmunoprecipitation assay (RIPA) buffer $(150 \mathrm{~mm} \mathrm{NaCl}, 10 \mathrm{~mm}$ Tris- $\mathrm{HCl}(\mathrm{pH}$ 7.5), $1 \%$ Nonidet P-40, $0.1 \%$ sodium dodecyl sulfate (SDS), and $0.2 \%$ sodium deoxycholate) containing Complete Protease Inhibitor Cocktail (Roche Applied Science, Germany).

Immunoprecipitation and Immunoblotting To detect the ubiquitination of Herp, HEK293 cells transfected with pcDNA3.1 (mock), pcDNA3.1-Herp-FLAG, pcDNA3.1-Herp9KR-FLAG, pcDNA3.1- $\Delta$ Ub-Herp-FLAG, or pcDNA3.1- $\Delta$ Ub-Herp4KR-FLAG were lysed and their proteins were solubilized on ice in RIPA buffer containing a protease inhibitor mixture. The lysates were subjected to immunoprecipitation overnight with primary antibodies or normal immunoglobulin G (IgG) (Santa Cruz Biotechnology, Inc.). Antibody-bound complexes were isolated by incubation with protein $\mathrm{G}$-Sepharose beads for $4 \mathrm{~h}$ before being washed in RIPA buffer. The protein complexes were eluted in $2 \times$ SDS sample buffer and analyzed by Western blotting. ${ }^{17)}$

Densitometric and Statistical Analysis Band intensities from western blots were densitometrically quantified using an LAS-3000 luminescent image analyzer (FUJI Photo Film Co., Ltd., Tokyo, Japan). Statistical analysis was performed using Student's $t$-test.

\section{RESULTS}

Accumulation of Herp in Synoviolin-Null Cells In previous studies, endogenous Herp levels were found to be 3.6 times higher in synoviolin-null cells than in wt cells. ${ }^{13)}$ In contrast, quantitative PCR revealed no significant differences in Herp mRNA expression between these two cell groups, which also exhibited similar levels of the ER protein BiP (Supplementary Figs. 1A, B). These results suggested that the accumulation of Herp was due to defects in the protein degradation pathway. To further investigate this, we next compared Herp degradation over time in synoviolin-null and wt cells. As shown in Fig. 1A, after treatment with cycloheximide, a protein synthesis inhibitor, the degradation of endogenous Herp in synoviolin-null cells was delayed compared with that in wt cells. Furthermore, Herp was found to accumulate in cycloheximide-treated cells after administration of the proteasome inhibitors MG132 and lactacystin (Fig. 1B, Supplementary Fig. 1C). The effects of MG132 and lactacystin on Herp stability were also observed in synoviolin-null cells, suggesting that synoviolin-independent pathways are also involved in the degradation of Herp.

Effects of Synoviolin on Herp Stability To investigate the effect of synoviolin on Herp stability, we overexpressed synoviolin and its mutant forms together with Herp in 
HEK293 cells. Herp level was decreased by exogenous expression of synoviolin, but not by expression of a synoviolin C307A mutant lacking E3 ubiquitin ligase activity, ${ }^{18)}$ indicating that the lack of E3 ubiquitin ligase activity caused the accumulation of Herp (Fig. 2). We expect that this occurred because the C307A mutant inhibited endogenous synoviolinmediated ubiquitination in a dominant-negative manner, as reported previously. ${ }^{18)}$ In addition, we investigated the effects of synoviolin overexpression on the ubiquitination of Herp. We found that the proportion of ubiquitinated Herp to non-ubiquitinated Herp was higher in HEK293 cells overexpressing synoviolin than in the empty vector control (Supplementary Fig. 2). Therefore, Herp is likely a substrate of synoviolin. Taken together, these results indicate that Herp is in part degraded by the synoviolin-dependent proteasome.
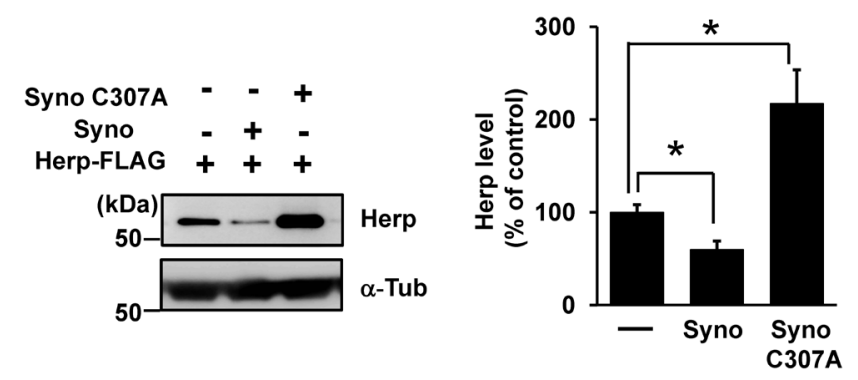

Fig. 2. Effects of Synoviolin Overexpression on Herp Stability

Western blot analysis of lysates prepared from HEK293 cells overexpressing pcDNA (-), pcDNA-Syvn (Syno), or pcDNA-Syvn C307A (Syno C307A), together with pcDNA-Herp-FLAG and pcDNA-ubiquitin-HA, and treated with $20 \mu \mathrm{g} / \mathrm{mL}$ cycloheximide and $10 \mu \mathrm{M}$ MG132 for $6 \mathrm{~h}$. Herp in RIPA-solubilized lysates $(20 \mu \mathrm{g})$ was detected by immunoblotting using an anti-Herp antibody. $\alpha$-Tubulin $(\alpha$-Tub) levels in the lysates were used as an internal loading control. Herp levels were quantified from three independent experiments. Values are expressed as the mean \pm S.E. $* p<0.05$ (Student's $t$-test), pcDNA vs. pcDNA-Syno or pcDNA-Syno C307A.
Proteasome Degradation and Ubiquitination of Herp To determine whether the degradation of Herp via synoviolin was mediated by the ubiquitin-dependent proteasomal path-

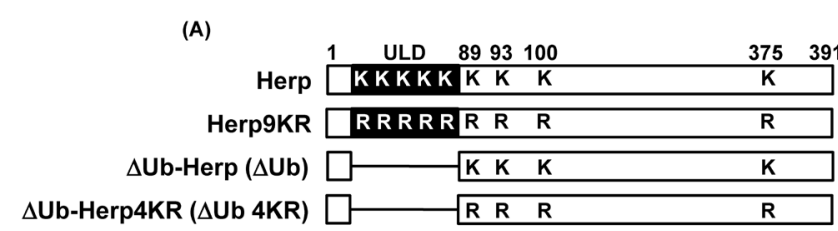

(B)

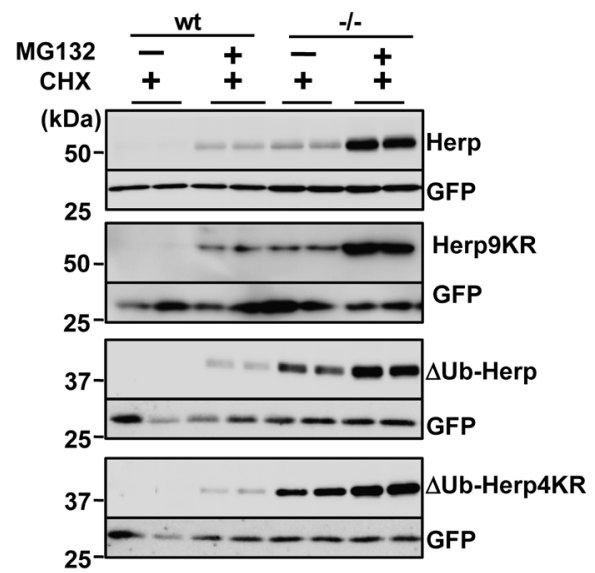

Fig. 3. Effect of Lysine Residues on Herp Degradation

(A) Schematic diagram of Herp wild-type and mutant proteins with the positions of all lysine residues indicated. ULD, ubiquitin-like domain. (B) Herp wild-type and mutant proteins were expressed in synoviolin-null $(-/-)$ and wild-type (wt) cells, together with green fluorescent protein (GFP), by transduction of expression vectors. Cells were treated with $20 \mu \mathrm{g} / \mathrm{mL}$ cycloheximide and $10 \mu \mathrm{M}$ MG132 for $6 \mathrm{~h}$. Herp wild-type and mutant proteins were then detected in RIPA-solubilized lysates $(20 \mu \mathrm{g})$ by immunoblotting using an anti-Herp antibody. GFP levels in the lysates were used as an internal loading control. CHX, cycloheximide. Each sample was run in duplicate.

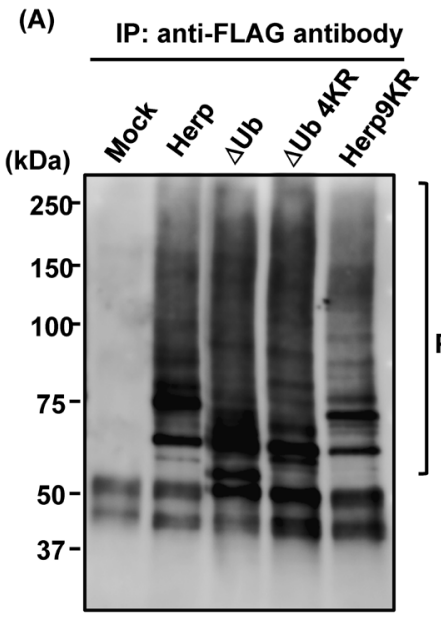

IB: anti-Ub antibody

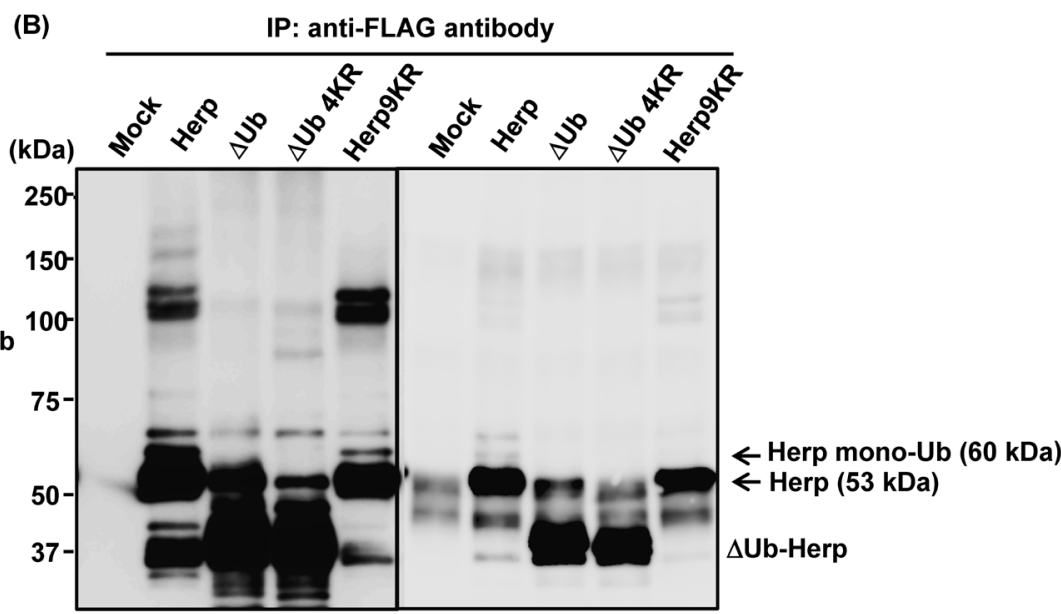

IB: anti-Herp antibody IB: anti-FLAG antibody

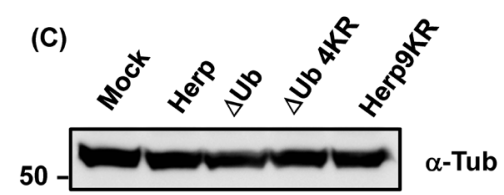

Fig. 4. Detection of Herp Ubiquitination

(A) To detect ubiquitination of wild-type Herp, Herp9KR, $\triangle$ Ub-Herp, and $\Delta$ Ub-Herp4KR, these proteins, each carrying a FLAG tag, were separately overexpressed in HEK293 cells and immunoprecipitated using an anti-FLAG antibody. The immunoprecipitates were then analyzed by Western blotting using an anti-ubiquitin antibody. (B) Levels of wild-type Herp, Herp9KR, $\Delta$ Ub-Herp, and $\Delta$ Ub-Herp4KR in each immunoprecipitate were confirmed using an anti-FLAG antibody. (C) $\alpha$-Tubulin ( $\alpha$-Tub) levels in the lysates were used as an internal loading control. IP, immunoprecipitation; IB, immunoblot. 
way, we used the proteasome inhibitor MG132 to investigate the stability of this protein and mutant forms lacking the ULD or in which all nine lysine residues had been substituted with arginine (Fig. 3A). MG132 inhibited the degradation of Herp and mutant Herp proteins in wt and synoviolin-null cycloheximide-treated cells (Fig. 3B), suggesting that Herp was degraded via the proteasome system. To examine whether the lysine residues of Herp were required for its ubiquitination, we next investigated the extent to which Herp and mutant Herp were ubiquitinated. MG132-treated cells overexpressing HerpFLAG or mutant Herp-FLAG were subjected to immunoprecipitation with an anti-FLAG antibody, and ubiquitination of the isolated Herp proteins was subsequently determined by Western blotting with an anti-ubiquitin antibody. Although levels of polyubiquitinated Herp9KR-FLAG were lower than those of polyubiquitinated Herp-FLAG (Fig. 4A), smearing indicative of ubiquitination was observed in blots of Herp9KRFLAG samples. Moreover, the extent of $\Delta \mathrm{Ub}$-Herp and $\triangle$ Ub-Herp4KR-FLAG ubiquitination was similar to that of Herp-FLAG ubiquitination, consistent with previous results. $\left.{ }^{6}\right)$ Therefore, these findings suggest that amino acids other than lysine were involved in Herp ubiquitination.

\section{DISCUSSION}

In this study, we showed that synoviolin is involved in the intracellular degradation of Herp. In contrast, synoviolin did not induce degradation of BiP, which, similar to Herp, is an ER protein and key ER stress-inducible regulatory component of ERAD. In addition, synoviolin appears to preferentially target Herp for degradation, as Herp accumulated in synoviolinnull cells, and expression of dominant-negative mutant synoviolin lacking E3 ubiquitin ligase activity resulted in greater accumulation of Herp than the empty vector control.

In this study, the degradation of Herp was found to be regulated by lysine-independent proteasomal pathways. Ubiquitin ligase catalyzes the ubiquitination of lysine residues; however, our results showed that lysine ubiquitination was not required for Herp degradation. Herp has nine lysine residues, five of which are located in the ULD, with the remainder being downstream of this domain. Our previous results suggested that deletion of the Herp ULD enhances Herp stability but does not affect its polyubiquitination. ${ }^{6}$ Moreover, it has recently been reported that the ULD is essential for Herp degradation, and the lysine residue in this domain at position 61 is the site of gp78-mediated polyubiquitination. ${ }^{19)}$ Consistent with this finding, we demonstrated that Herp9KR, which contained none of the lysine residues present in the wild-type Herp sequence, was degraded. However, substitution of all nine lysine residues within Herp did not completely inhibit its degradation; therefore, other degradation pathways were considered to be involved. Recently, N-terminal or non-lysinemediated ubiquitination mechanisms have been described, in which serine or threonine residues are sufficient to induce ubiquitination and protein degradation. ${ }^{20,21)}$ Although we made a preliminary attempt to identify ubiquitinated sites in Herp using LC-MS/MS analysis of the $60-\mathrm{kDa}$ band, which may comprise Herp $(53 \mathrm{kDa})$ and ubiquitin $(7 \mathrm{kDa})$, shown in Fig. $4 \mathrm{~B}$, ubiquitination was not detected. However, Herp itself, including the 101-126 amino acid region, would be expected to yield this band. Moreover, ubiquitination of $\Delta \mathrm{Ub}$-Herp has been observed, ${ }^{6)}$ and the ubiquitination sites are considered to be present in regions other than the ULD (the 10-88 amino acid region). Further studies will be required to determine whether N-terminal or non-lysine-mediated ubiquitination is involved in Herp degradation.

Alternatively, the ubiquitin-independent proteasomal pathway may be implicated in Herp degradation. Two pathways are known to induce proteasomal degradation. The most commonly studied of these pathways involves targeting of polyubiquitinated proteins to the $26 \mathrm{~S}$ proteasome. In contrast, few proteins are degraded by the $20 \mathrm{~S}$ proteasome. NQO1 associates with the $20 \mathrm{~S}$ proteasome and prevents degradation of short-lived proteins, such as $\mathrm{p} 53,{ }^{22)} \mathrm{c}-\mathrm{Fos},{ }^{23)}$ and the initiation factor eIF4GI. ${ }^{24)}$ Our own recent work has identified a role for the ubiquitin-independent $20 \mathrm{~S}$ proteasomal pathway in Herp degradation. ${ }^{13)}$

In conclusion, this study showed that Herp stability was regulated by synoviolin through lysine ubiquitination-independent proteasomal degradation.

Acknowledgments We thank Dr. Koichi Kokame (National Cerebral and Cardiovascular Center) for providing the anti-Herp antibody. This work was supported by a Grant-inAid for Scientific Research (C) 26460100 (to T.M.), a Grantin-Aid for the Strategic Medical Science Research Center (The MIAST Project) (to H.K.), a Grant-in-Aid for Strategic Medical Science Research S1491001 (to H.K.) from the Ministry of Education, Culture, Sports, Science and Technology of Japan, and a Grant from the Takeda Science Foundation (to T.M.).

Conflict of Interest The authors declare no conflict of interest.

Supplementary Materials The online version of this article contains supplementary materials.

\section{REFERENCES}

1) Kokame K, Agarwala KL, Kato H, Miyata T. Herp, a new ubiquitin-like membrane protein induced by endoplasmic reticulum stress. J. Biol. Chem., 275, 32846-32853 (2000).

2) Schulze A, Standera S, Buerger E, Kikkert M, van Voorden S, Wiertz E, Koning F, Kloetzel PM, Seeger M. The ubiquitin-domain protein HERP forms a complex with components of the endoplasmic reticulum associated degradation pathway. J. Mol. Biol., 354, 1021-1027 (2005).

3) Hori O, Ichinoda F, Yamaguchi A, Tamatani T, Taniguchi M, Koyama Y, Katayama T, Tohyama M, Stern DM, Ozawa K, Kitao Y, Ogawa S. Role of Herp in the endoplasmic reticulum stress response. Genes Cells, 9, 457-469 (2004).

4) Okuda-Shimizu Y, Hendershot LM. Characterization of an ERAD pathway for nonglycosylated $\mathrm{BiP}$ substrates, which require Herp. Mol. Cell, 28, 544-554 (2007).

5) Sato T, Sako Y, Sho M, Momohara M, Suico MA, Shuto T, Nishitoh H, Okiyoneda T, Kokame K, Kaneko M, Taura M, Miyata M, Chosa K, Koga T, Morino-Koga S, Wada I, Kai H. STT3B-dependent posttranslational $N$-glycosylation as a surveillance system for secretory protein. Mol. Cell, 47, 99-110 (2012).

6) Sai X, Kokame K, Shiraishi H, Kawamura Y, Miyata T, Yanagisawa $\mathrm{K}$, Komano H. The ubiquitin-like domain of Herp is involved in Herp degradation, but not necessary for its enhancement of amyloid beta-protein generation. FEBS Lett., 553, 151-156 (2003).

7) Marutani T, Maeda T, Tanabe C, Zou K, Araki W, Kokame K, 
Michikawa M, Komano H. ER-stress-inducible protein, Herp, facilitate the degradation of $\gamma$-secretase cofactors. Biochim. Biophys. Acta, 1810, 790-798 (2011).

8) Kim TY, Kim E, Yoon SK, Yoon JB. Herp enhances ER-associated protein degradation by recruiting ubiquilins. Biochem. Biophys. Res. Commun., 369, 741-746 (2008).

9) Mukhopadhyay D, Riezman H. Proteasome-independent functions of ubiquitin in endocytosis and signaling. Science, 315, 201-205 (2007).

10) Amano T, Yamasaki S, Yagishita N, Tsuchimochi K, Shin H, Kawahara K, Aratani S, Fujita H, Zhang L, Ikeda R, Fujii R, Miura N, Komiya S, Nishioka K, Maruyama I, Fukamizu A, Nakajima T. Synoviolin/Hrd1, an E3 ubiquitin ligase, as a novel pathogenic factor for arthropathy. Genes Dev., 17, 2436-2449 (2003).

11) Wu T, Zhao F, Gao B, Tan C, Yagishita N, Nakajima T, Wong PK, Chapman E, Fang D, Zhang DD. Hrd1 suppresses Nrf2-mediated cellular protection during liver cirrhosis. Genes Dev., 28, 708-722 (2014).

12) Yamasaki S, Yagishita N, Sasaki T, Nakazawa M, Kato Y, Yamadera T, Bae E, Toriyama S, Ikeda R, Zhang L, Fujitani K, Yoo E, Tsuchimochi K, Ohta T, Araya N, Fujita H, Aratani S, Eguchi K, Komiya S, Maruyama I, Higashi N, Sato M, Senoo H, Ochi T, Yokoyama S, Amano T, Kim J, Gay S, Fukamizu A, Nishioka K, Tanaka K, Nakajima T. Cytoplasmic destruction of p53 by the endoplasmic reticulum-resident ubiquitin ligase 'Synoviolin.' $E M B O \mathrm{~J}$, 26, 113-122 (2007).

13) Maeda T, Tanabe-Fujimura C, Fujita $Y$, Abe C, Nanakida Y, Zou K, Liu J, Liu S, Nakajima T, Komano H. NAD(P)H quinone oxidoreductase 1 inhibits the proteasomal degradation of homocysteineinduced endoplasmic reticulum protein. Biochem. Biophys. Res. Commun., 473, 1276-1280 (2016).

14) Kny M, Standera S, Hartmann-Petersen R, Kloetzel PM, Seeger M. Herp regulates Hrd1-mediated ubiquitylation in a ubiquitin-like domain-dependent manner. J. Biol. Chem., 286, 5151-5156 (2011).

15) Yagishita N, Ohneda K, Amano T, Yamasaki S, Sugiura A, Tsuchimochi K, Shin H, Kawahara K, Ohneda O, Ohta T, Tanaka S,
Yamamoto M, Maruyama I, Nishioka K, Fukamizu A, Nakajima T. Essential role of synoviolin in embryogenesis. J. Biol. Chem., 280, 7909-7916 (2005).

16) Maeda T, Marutani T, Zou K, Araki W, Tanabe C, Yagishita N, Yamano Y, Amano T, Michikawa M, Nakajima T, Komano H. An E3 ubiquitin ligase, Synoviolin, is involved in the degradation of immature nicastrin, and regulates the production of amyloid betaprotein. FEBS J., 276, 5832-5840 (2009).

17) Tanabe C, Maeda T, Zou K, Liu J, Liu S, Nakajima T, Komano H. The ubiquitin ligase synoviolin up-regulates amyloid beta production by targeting a negative regulator of gamma-secretase. J. Biol. Chem., 287, 44203-44211 (2012).

18) Gao B, Lee SM, Chen A, Zhang J, Zhang DD, Kannan K, Ortmann RA, Fang D. Synoviolin promotes IRE1 ubiquitination and degradation in synovial fibroblasts from mice with collagen-induced arthritis. EMBO Rep., 9, 480-485 (2008).

19) Yan L, Liu W, Zhang H, Liu C, Shang Y, Ye Y, Zhang X, Li W. Ube2g2-gp78-mediated HERP polyubiquitylation is involved in ER stress recovery. J. Cell Sci., 127, 1417-1427 (2014).

20) Cadwell K, Coscoy L. Ubiquitination on nonlysine residues by a viral E3 ubiquitin ligase. Science, 309, 127-130 (2005).

21) Wang X, Herr RA, Chua WJ, Lybarger L, Wiertz EJ, Hansen TH. Ubiquitination of serine, threonine, or lysine residues on the cytoplasmic tail can induce ERAD of MHC-I by viral E3 ligase mK3. J. Cell Biol., 177, 613-624 (2007).

22) Asher G, Lotem J, Cohen B, Sachs L, Shaul Y. Regulation of p53 stability and p53-dependent apoptosis by NADH quinone oxidoreductase 1. Proc. Natl. Acad. Sci. U.S.A., 98, 1188-1193 (2001).

23) Adler J, Reuven N, Kahana C, Shaul Y. c-Fos proteasomal degradation is activated by a default mechanism, and its regulation by $\mathrm{NAD}(\mathrm{P}) \mathrm{H}$ :quinone oxidoreductase 1 determines c-Fos serum response kinetics. Mol. Cell. Biol., 30, 3767-3778 (2010).

24) Alard A, Fabre B, Anesia R, Marboeuf C, Pierre P, Susini C, Bousquet $\mathrm{C}$, Pyronnet $\mathrm{S}$. NAD $(\mathrm{P}) \mathrm{H}$ quinone-oxydoreductase 1 protects eukaryotic translation initiation factor 4GI from degradation by the proteasome. Mol. Cell. Biol., 30, 1097-1105 (2010). 